\title{
Design and Implementation of a Secured Web based Medical Record Management System: A Case Study of Federal University Wukari (FUW) Clinic
}

\author{
Bitrus Joseph \\ Department of Computer Science, \\ Federal University Wukari, \\ P.M.B.1020, Katsina- Ala Road \\ Wukari, Taraba State, Nigeria
}

\author{
Wadzani A. Gadzama \\ Information \& Communication \\ Technology Unit \\ Federal Polytechnic Mubi, P.M.B. \\ 35 Mubi, Adamawa State, Nigeria
}

\author{
Ngubdo Maigana A. \\ Department of Computer \\ Engineering \\ Federal Polytechnic Mubi,
}

\author{
Agu E. O. \\ Department of Computer Science, \\ Federal University Wukari, P.M.B.1020, \\ Katsina- Ala Road Wukari, Taraba State, Nigeria
}

\begin{abstract}
Patient records constitute the bulk of the medical records of almost all the health care centers all over the world; The existing system of medical record keeping used in FUW Clinic is predominantly paper-based and it is associated with problems such as misplacement of patients' record, unnecessary duplication of patients' record as well as lack of effective back up facilities. In an attempt to address the problems associated with paper-based medical record, this project aimed at automating the whole processes by designing a web-based application to minimize the cost of procuring stationery materials needed for paper-based record keeping and enhancing the integrity and security of the patients' medical records. The proposed system uses the following security tools to prevent unauthorized users from gaining access to the system resources: The sign up module, the security measure employed on the sign up module to ensure that no unauthorized person is allowed to create a user account is that when a user clicks on the sign up button a prompt will be displayed asking the user to enter a One Time PIN (OTP) which is only obtainable from the appropriate authorities of the clinic. Another security tool used is the encryption of user's password on the login tables using the MD5 encryption function, once a user submits a new user account details during the sign up processes, the user's password will be posted to the login table in an encrypted format. To achieve these objectives, the technologies used in the development of this automated system were: HTML, CSS, Javascript and PHP as the Scripting languages, MySQL as the database engine. The system grants different users privileges based on their statutory functions in the clinic which allow the clinical staff (users) the view and perform actions strictly within the domain of their official duties.
\end{abstract}

\section{General Terms}

Electronic Medical Record (EMR) Systems

\section{Keywords}

FUW Clinic, MD5 encryption, Medical Records, Patients

\section{INTRODUCTION}

The patient medical record is a detailed account of patient's health and disease after he/she has sought medical help from a health care center; usually, the notes in the record are made by nurses, laboratory technicians or the physicians. The patient's record contains findings, considerations, test results and treatment information related to the disease that the patient might be suffering from. Medical records are usually kept confidential in an attempt to protect the patient's privacy.

Presently the medical record facilities at the Federal University Wukari (FUW) clinic involve the use of a paperbased patient folder that contains all the relevant medical data about a particular patient; the manual or paper based record management system is associated with problems which include but not limited to inefficiency, inaccuracy, time consumption, inconveniency, laborious and sluggish. It is against this background that the need for automating the existing manual systems arises [1].

The Electronic Medical Record (EMR) management system may contain some elements of paper record for example, in the event that a doctor wanted to refer a patient to another clinic or a hospital, the diagnostic information and clinical notes needs to be printed or handwriting on a paper and then sent to the new doctor to be involved for further analysis and examination. In the present era of globalization and advancement in information and communication technology, the need for efficient record keeping increases because of its importance in decision making which makes life better for man; health they say is wealth, therefore there is a need for improving the quality of healthcare delivery. In a recent study on design and implementation of hospital management system, [2] pointed out that, the advancement in technology and globalization remains an active ingredient that enhances the quality of healthcare delivery provided by medical institutions as a result of improvement in the quality of medical services rendered by the various medical institutions all over the world.

The emergence of computer technology has affected the medical institutions, like most other industries all over the world. The application of computers on the delivery of health care services has become a common place practice. In some cases, it involves applying certain technologies to convert the existing paper documents into an electronic format. In others, it is a transition towards the use of the EMR. The EMR is designed to replace the paper record as the primary format of record keeping in the various healthcare centers across the globe [3]. 


\subsection{Statement of the Problem}

The under-listed points constitute the major problems of the existing medical record system in FUW clinic as identified by this project:

i. Inefficiency of the existing System: The use of paper for keeping all the medical records of the clinic have reduced the efficiency of the existing system for example, various changes to medical records like staff details are difficult to make as paper work is involved [4].

ii. Insecurity of data: The patient folders and medical cards used for record keeping is easily exposed to unauthorized users; which can easily get vital patient's information from the clinic because the patient folder which contains a patient's medical records are just kept on the shelf in a file cabinet without any security lock [5,2].

iii. Time consuming: By using medical cards, considerable amount of times are wasted when the medical cards needs to pass from the nurse to the doctor and then to pharmacy for drug collection; also, some of the clinic staff needs to spend sometimes to organize the medical cards from time to time [4].

iv. Lack of effective back up facilities: lack of effective back up facilities for data could lead to loss of the entire information that is kept in the clinic, in the event of disaster [6].

\section{LITERATURE REVIEW}

The major advantage of adopting the electronic-based patient records is its vast possibility of strengthening the linkage between the clinical records on one hand and management information system on the other hand so that resources used and quality of medical services rendered can be analyzed using clinical data. Directors and top administrative officers of medical institutions like other industries tends to benefit from the automation of their organizational record keeping; because such records can serve as an effective tool for managerial decision making [5]. A recent study by [7] indicated that medical records are confidential information kept for each patient by health care professionals or organizations that contains the patient's personal details such as name, residential address, and date of birth, a summary of the patient's medical history and documentation of each event including symptoms, diagnosis, and treatment; it also contains a report of the results of a medical examination of a patient.

\subsection{Overview of Paper-based Medical Record Systems}

Many of the clinical notes and management information that flow within clinics are predominantly paper-based. Using paper-based record management system is not very reliable. This is based on the fact that papers can damage easily and cannot be kept for a very long period of time. There could be issues of data anomaly because data errors are not easy to be detected. Furthermore the use of paper-based medical record system can be seen as a yesterday's technique or obsolete technique as far as medical records is concerned because of the availability of new technology as well as improvement in technology that are widely employed in medical settings all over the world [8]

Presently, the FUW clinic like many other clinics still keep her medical record manually, one notable technique used is outpatient medical card. Medical cards are a printed card which comprises brief patient information, the date for each visit to the clinic, diagnosis, clinical notes and treatment for each diagnosis. A medical card will be generated by the nurse or a receptionist when the patient first visits a clinic. Usually patient will be asked by the nurse/receptionist to supply some vital and personal information like the nature of patient's problem, how long the patient has been suffering from the problem (sickness) in question, occupation of the patient, phone number, and residential address are to be manually written on the medical card during the registration. After that, the medical card will be passed to the doctor to write down the diagnosis and treatment after the doctor has diagnosed the patient and passed it back to the dispensary or pharmacy. The pharmacist will prepare the medicine based on the prescription written on the medical card. Finally, after getting their medicine, the patient will leave. The medical card is mainly used for recording the diagnosis and treatment that have been done on the patient [9].

\subsection{Review of Web-based and Electronic Medical Record Management Systems}

A recent study by [10] demonstrated that nowadays, in our day to day activities everything around us is going for digitization. The emergence of web application domains have helped to minimize and reduce the real-world problem. The electronic health records offer the medical institutions and organizations the possibility to improve the management of their patient's medical records. Most of the healthcare software development organizations work in isolation from the healthcare providers which leads to complications and confusions with regard to correctness and interoperability among different healthcare systems. The term interoperability refers to the way in which different subsystems access and use the data reliably and quickly from various sources without the occurrence of errors. The interoperability among the healthcare information systems enables these systems to communicate with each other in order to share information, improving its high availability. For example, a user whose computer system runs on a Unix operating system and another user whose computer is running on the Windows operating system can all access the web-based system regardless of the differences in hardware design.

A recent study conducted by [11] defines an electronic medical record as a digital version of a traditional paper-based medical record of a patient; an electronic medical record system can be implemented as desktop based application, mobile based or web-based depending on the end user's requirement. The Electronic Medical Record can be designed to work with computer systems (both laptops and desktops); it can also be designed to work with the handheld mobile devices such as the Android devices. The use of electronic medical record system minimizes the amount of physical storage space required for storing patient and staff records, and they also allow the patient records to be shared by the staff of the various departments of the clinic and administrative officers without physically transporting the records because they are readily accessible at any time from the clinic's database using computer devices. Time and distance being the major barriers to the retrieval of information from the clinic's medical record in the case of paper-based medical record has been addressed by the introduction and implementation of web-based version of the EMR.

A recent study by [12] on Hospital Information Systems in Nigeria reveals that the methods used in collection, 
processing, and storage of patient information that helps in administering clinical treatment to patient dates back to the origin of medicine. The technique used in the collection of patients' records and the ways in which this information is used and subsequently stored for future references has continued to evolve from regular paper note takings to electronic hospital information systems. The electronic hospital or clinical record system makes use of the computer technology to record and store patient's record on dedicate web servers instead of the traditional paper record keeping system.

The increasing demand for well-structured and accessible patient data, in accordance with development in computer science has triggered a great interest in the development of electronic patient record. Computers have the potential to improve legibility, accessibility and structure of the patient's record. The performance of healthcare system can be improved greatly with the use of computers and modern information technology. The healthcare system and medical institutions in the developing and third world countries like Bangladesh, India, Myanmar, and Nigeria among others did not fully utilize the power of computers, process automation, and information technology yet. In those countries, the medical services did not completely integrate computers and information technology into their day to day activities [13]. The computer- based medical record minimizes the amount of physical storage space required for storing patient and staff records, and they also allow the records to be shared by the staff of the various departments of the clinic and administrative officers without physically transporting the records because they are readily accessible at any time from the clinic's website (proposed system). Time and distance being the major barriers to the retrieval of information from the clinic's medical record (paper-based record) has been addressed by the introduction and implementation of a computerized medical record system.

\subsection{Benefits of Web-based Medical Record systems}

A recent research conducted by [14] noted that over the years, there have been issues concerning fast and easy hospital management that ranges from managing healthcare quality improvement, compliance record keeping and documentation, workflow and analytics, and patient status management functionality which is aimed at improving the services rendered to patients that visit the hospital, there is need for application that addresses the administrative needs of healthcare practices, ambulatory surgery centers, surgical hospitals and clinics; the application that can address all the needs mentioned above is usually developed using computer technologies based on the fact that computers have improved the accuracy, speed and reliability of many of the administrative and technical tasks traditionally involved in patient care besides improving the services offered to patients. [14] also opined that Web-based hospital management information systems (a variant of the secured web-based medical record management system developed by this project) contain strategic decision support systems and clinical record keeping and documentation systems. Some of the clinical support systems include Laboratory Information Systems (LIS), Radiology Information Systems (RIS), Computerized Order Entry (COE) and Pharmacy Information Systems (PIS).

The increased usage of telecommunication facilities like desktop computers and laptop computer systems in medical industry and the development of hospital information systems improve workflow and increase patients' access to health care; it also makes consultation and timely treatments of patients easier because it increases the productivity of the medical personnel $[12,16]$. According to [17], computer technologies assists doctors, nurses and other role players in the health care industries do their jobs more quickly and very accurately. In hospitals and clinics nowadays, computers keep track of patient's records, maintain appointment and schedule operations, place an order for supplies of hospital and clinical equipment and drugs. The health related data for a particular patient are stored using computers as it offers an excellent means of data storage with optimum efficiency and data security; the points above leads to a need for the design and implementation of a secured web-based medical record management system in Federal University Wukari clinic.

A recent study by [6] stressed that the design and implementation of online outpatient database system provides a facility for online data storage, online updating of data as well as online data retrieval. This system makes a commitment toward very limited use of paper work and also helps the Doctors and other staff of the clinic to discharge their duties faster and more accurate. This system stores all the patient data, staff data and other related medical records electronically with minimal amount of paper usage; information can be retrieved very easily without physically searching the various registers from the various departments of the clinic.

Modern information technology is being increasingly used in the healthcare sector with the sole objective of enhancing the availability of improved medical services at a reduced cost. High quality healthcare depends on extensive and carefully planned information processing. The current development of cloud computing in the healthcare context will have a big impact on the healthcare sector [15]. The proposed system will offer all the benefits listed above with regard to information storage, retrieval and updating as well as increasing the speed and efficiency of clinicians in discharging their duties in FUW clinic.

\subsection{Review of Security Issues in Web-based Medical Record Systems}

Security and privacy are two important issues in the protection of health information. The aim is to keep the medical privacy of confidential information about the patient. Privacy must be protected in the business of medical information technology systems. The concept of security in a medical setting is a very sensitive issue because of the confidential nature of medical records. Encryption technique is well defined to offer appropriate solutions of protection for privacy and security of patient health records [18]. The term security as used in the context of this project work connotes the measures taken as a precaution against theft or unauthorized users. It can also be regarded as actions taken to prevent unauthorized users from gaining access to FUW clinic medical records. Security is a term that describes the state of being free from risk or danger. The need for safety is fundamental to human existence and this applies to almost all the sphere of human life. Even though the purpose of a network is to share resources, an unfortunate side effect emerging from the rapidly growing usage of a network of computer devices is that the safety of our personal information stored and/or transferred on such devices is at risk. The more the world becomes connected (Either through the intranet that is internal to an organization or global connection using the internet), the more security becomes an issue. However, because of the high level of confidentiality of medical records, coupled with an effort to maintain patients' 
privacy and data security, computerized medical records need to be securely transmitted through networks. Most patients feel that their health information should be kept private and protected from unauthorized users. Over the years, security concerns for electronic medical records have been on the increase; hence a few possible techniques that can be built into EMR systems in order to improve the level of security and limit unauthorized accesses may include the following:

i. Use of access control tools like passwords

ii. Use of Personal Identification Numbers (PIN) during a user account creation

iii. Encrypting the stored clinical information according to [19].

The tools identified above have been widely used in securing a reasonable number of web applications across the globe. According to [18] the security threats to health records can be classified under three headings: (1) human threats, such as from hackers. (2) Natural and environmental disasters, such as flood, earthquakes, hurricanes and fires; and (3) Technology failures such as computer system crashes. A disaster recovery planning can protect a health record that is a series of steps to restore and protect the information technology infrastructure in the event of a disaster. Paying attention to cyber security can prevent hacker's attack through internet. A healthcare organization can maintain the security of its medical records by adopting the appropriate Cyber security standards which enables them to practice safe security techniques to stop cyber-attack.

\subsection{Review Summary and Research Gap}

Having reviewed some scholarly articles and project works that are relevant to medical records at both Paper-based and electronic based levels, the need for electronic medical record management systems and security issues in electronic medical record systems, this project has come up with the following issues that have not been addressed properly by the reviewed articles:

i. In respect to concurrent access to medical records by the clinicians, some of the papers reviewed in this project ended up in developing a single user desktop application for their project, which can only be operated by a single user at a time and does not support a concurrent access to medical data of any given medical institution. In an effort to provide a system that offers a concurrent access to medical records, this project work is determined to develop a distributed web-based application that can be accessed concurrently by multiple users at the same time.

ii. None of the papers reviewed in this project have taken the testing of the software developed into consideration, on the contrary this project have taken an in depth coverage of software testing.

\section{METHODOLOGY}

Methodology refers to the description of methods chosen to achieve the objectives of the proposed system; these methods are made up of controlled procedures and activities for generating a set of models that describe various aspects of a software system under development. The iterative software models have been adopted in this project. This project was developed using the combination of the under-listed scripting languages, software application and hardware components which are mostly suited for the development of similar software product like the secured web-based medical records management system. These tools includes: - HTML, CSS and JavaScript (for front end application design), MySQL as the database engine (back end database storage) and PHP for the operational logic. The following hardware components are used in the development of this project: Intel Pentium IV processor, $4 \mathrm{~GB}$ of RAM, 20GB of hard disk space, Keyboard, LCD monitor and Mouse

\section{RESULTS AND DISCUSSION}

This section presents the discussion of results achieved in this project. Some of the achievement made in the proposed system is the inclusion of the update patient profile module. This module enables an instant updating of a patient's basic bio-data information. Another achievement is the inclusion of the update staff profile module; this module enables the medical director to edit and update staff profile.

\subsection{Username and Password Authentication Using the Login Module}

This is the login page through which the administrator, doctors and specific users with valid login credentials can be granted access clinic forms. The login page will be displayed automatically when a user clicks on the login button. This form enables the user to submit their login credentials into the system; on submission of correct login credentials, a user will be granted an access to the system resources but when the login credentials are incorrect, the user is denied access to the system resources. A password reset option is also made available on the login form but it is strictly meant for staff password resetting. The result of username and password authentication using the login form is shown in figure 1 .

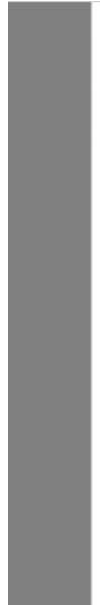

\section{FEDERAL UNIVERSITY WUKARI CLINIC}

Welcome, Please Login to Access the Clinic Resources

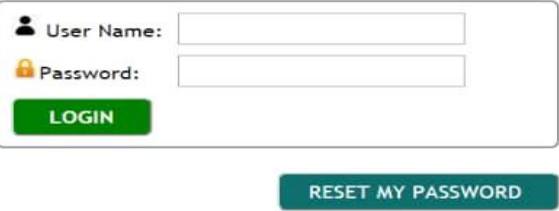

Figure 1: System login page 


\subsection{Patient Profile Module}

The patient bio-data records can be added by patients themselves or by the staff of the health records unit of the clinic. For a patient to upload his/her bio-data records, the patient has to sign up to create his/her user account. The new patient profile form contains the patient's basic bio-data information as shown in figure 2 .

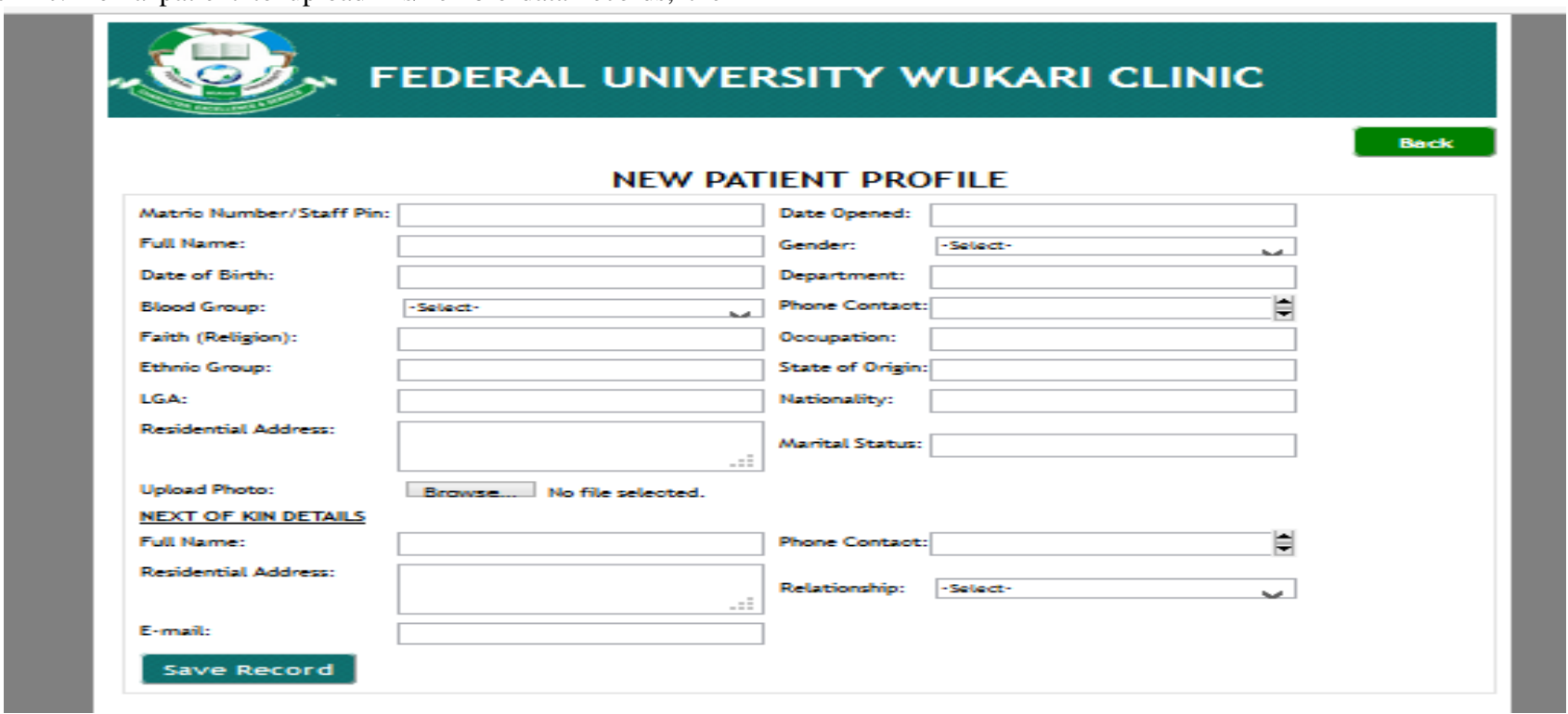

Figure 2: Implementation of the New Patient Profile Form

\subsection{Doctors' Module}

This is the page that will be displayed when a user logs in to the system as a doctor. This page grants a medical doctor an access to some list of activities such as adding inpatient consultation records, adding outpatient consultation records, adding inpatient treatment records, adding outpatient treatment records, adding death records. This module also enables the doctors to view the various records stored on the clinic database by the medical doctors. The doctors' module is shown in figure 3 .

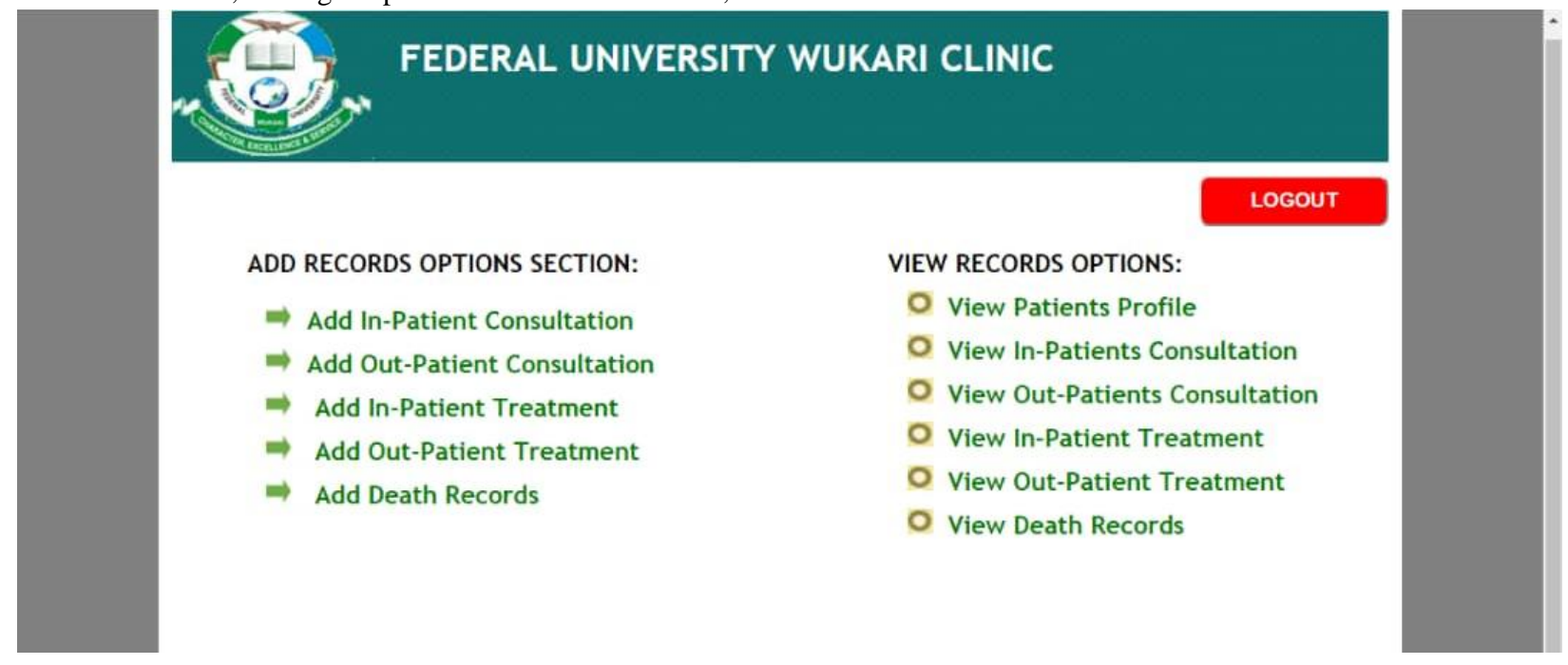

Figure 3: screen shoot of the Doctors' module

\subsection{Implementation of the Nurse Module}

The nurse module is the page that will be displayed when a staff logs in to the system as a nurse. This form gives a nurse an access to add some records into the database and also to view the records stored on the database from the nursing station of the clinic. The screen shoot of the nurse module is shown in figure 4 . 


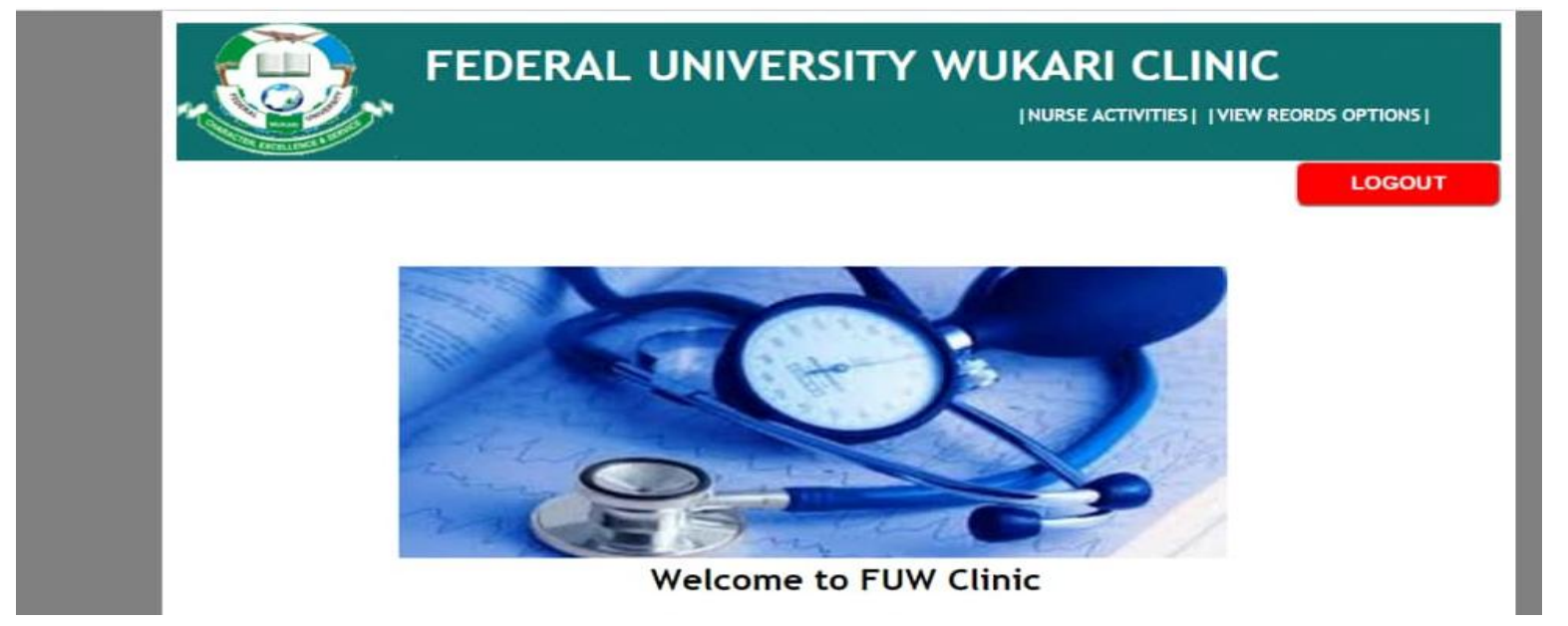

Figure 4: screen shoot of the nurse module

\subsection{Implementation of the add Death Record form}

This is the page that enables the medical doctors to add, store and view death records. The add death record form automatically loads once a medical doctor clicks on the add death record button. This form is used for saving or storing the death records into the death records table on the database. The implementation of the add death record form is shown in figure 5 .

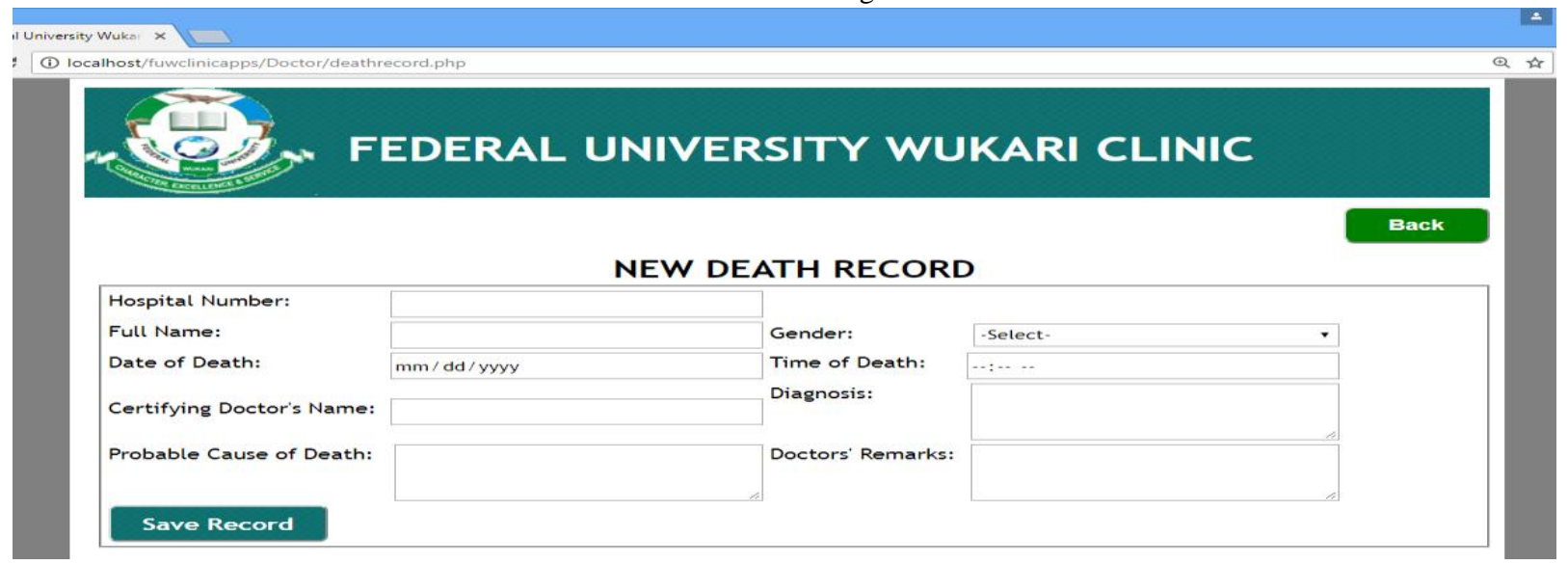

Figure 5: implementation of the add death record form.

\subsection{Implementation of the Secured Encrypted Users Password on the Login Tables.}

The login tables of the proposed system is implemented in such a way that once a new user has created a user account by signing up, the login credentials will be posted to the appropriate login table with the user's password encrypted. The encryption of a user's password is aimed at ensuring the security of a user's account; this is shown in the figure 6 .

\begin{tabular}{|c|c|c|c|c|c|}
\hline 10 & FullName & Email & Department & UserName & Password \\
\hline $12 \mathrm{Mr}$ & Abduham & haruna@gmail.com & Laboratory_Scientist & Abduhamid & 187ef4436122d1cc2f40dc2b92f0eba0 \\
\hline $13 \mathrm{Mr}$ & Mathias J & mathias123@gmail.com & Laboratory_Scientist & mathias & 590a84e4140f3457a7a626 \\
\hline & 然 & mercydo02@gmail.com & Laboratory_Scientist & mercy & bf2ff2ed3c83c3c5ce510c4666ffftbod \\
\hline
\end{tabular}

Figure 6: Implementation of the Secured Encrypted Users Password on the Login Tables.

\section{SUMMARY}

As far as this research project is concerned, patient records constitute the bulk of the medical records of all the health care centers all over the world; to this effect, medical records refers to the confidential information kept for each patient by heath care professionals or organizations that contains the patient's personal details such as name, residential address, and date of birth, a summary of the patient's medical history and documentation of each event including symptoms, diagnosis, treatment and outcome; it also contains a report of the results of a medical examination of a patient. 


\subsection{Suggestions for Further Work (Future Enhancements)}

In order to improve the proposed system in the future, it can be enhanced by including more features like doctor's appointment module, clinical inventory module and billing system that will be used in computing the cost of drugs ordered. The inclusion of such features into the proposed system will provide for more patient satisfaction and yield higher productivity of the health care workers.

\subsection{Recommendations}

Having seen the usefulness of the proposed project titled secured Web-based medical record management system to the Federal University Wukari clinic; I recommend the following to the clinic as stipulated below:

- The record keeping of the clinic should be completely automated.

- The staff of the various departments of the clinic should be adequately trained on how to use the proposed system.

- Appropriate policy guidelines should be formulated that will protect the privacy and security of patients' information.

\subsection{Conclusion}

Computing devices like the laptop computers and desktop computers has become one of the common means of accessing medical information within and outside the clinical settings. Special caution should be taken while using the computing devices for retrieval of the confidential medical records of a patient from the clinic's database by maintaining the security of Patients' medical records; this is achieved by restricting the access to a patient's medical data such that only authorized users can access the patient's records. The problem of manually searching the shelves of a file cabinet in order to locate a patient record has been addressed in this project by designing a secured web-based medical record management system.

\section{REFERENCES}

[1] Agu, E.O., Nwadialor, G.O., and Moses, T. 2016. Design and Implementation of a Dynamically Adapted Students' Admission System: African Journal of Education, Science and Technology Vol 3, 162-179

[2] Asabe, S. A., Monday, G., and Oye, N. D. 2013. Hospital Patient Database Management System: International Journal of Advanced Computer Technology, 2 (3)

[3] James, A. C. 2009. Computerization of Medical Records Retrieved on $18^{\text {th }}$ Jan, 2017 from http://www.ddclaw.com/news_events/articlesofinterest/p dfs/Computerization\%20of\%20Medical\%20Records.pdf.

[4] Adebisi, O.A, Oladosu, D.A, Busari, O.A and Oyewola, Y.V. 2015. Design and Implementation of Hospital Management System: International Journal of Engineering and Innovative Technology Volume 5(1)

[5] Wadzani, A. G. 2008. Computerized Medical Record System: A Case Study of F.U.T.Y Clinic. Unpublished Bachelor of Technology Research Project, Federal University of Technology Yola, Adamawa State

[6] Opeyemi, A.A., Blessing, O. A., and Blessing, E. O. 2016. An Online Outpatient Database System: A Case Study of General Hospital, Minna. Intelligent
Information Management, 103-114.

[7] Droma, F., Bulyaba, H., Ssebwato, J., Nakawooya, K., Musah, K. C., Ongoro, A., Collins, S., and Ndege, R. 2009. An Automated System for Patient Record Management: A Case study of St Francis Hospital Nsambya. Unpublished Bachelor of Information Technology Project Report, Makerere University

[8] Mohd-Zharif, A. R. 2010. Patient Registration System: Unpublished Bachelor of Computer Science Research Project, Universiti Tecknikal Malaysia Melaka.

[9] Yeo, Y. P. 2004. Clinical Management Systems: Unpublished Bachelor of Science (BSc) Project report, Universiti Malay

[10] Vinayak, S., Anant, G., Surendra, W., Eknath, S., and Bhadkumbh, S. M. 2016 WEB Based e-Health System and Services: International Journal of Innovative Research in Computer and Communication Engineering $4(3)$

[11] Daryl, A., Bulou, G., Nicholas, R., and Rohitash, C. 2015. Development of an Android Application for an Electronic Medical Record System in an Outpatient Environment for Healthcare in Fiji: Technical Report, AICRG, Software Foundation, Fiji, March 2015

[12] Ayodele, C. B. 2011. Hospital Information Systems in Nigeria: A Review of Literature. Journal of Global Health Care Systems 1(3)

[13] Biswas, S., Anisuzzaman, Tanjina, A., Kaiser, M. S., and Mamun, S. A. 2014. Cloud Based Healthcare Application Architecture and Electronic Medical Record Mining: An Integrated Approach to Improve Healthcare System: 2014 17th International Conference on Computer and Information Technology (ICCIT)

[14] Ilo, S.F., Igbajar, A., and Acholonu, J. C. 2015. Designing A Web Based Hospital Management System For MOUAU Clinic: International Journal of Trend in Research and Development, 2(6)

[15] Rabi, P. P., Manas, R. P., and Suresh, C. S. 2012. Design and Implementation of a Cloud based Rural Healthcare Information System Model: UNIASCIT, 2(1), 149-157

[16] Parameswari, R., and Prabakaran, N. 2013. An Android Enabled Mobile Cloud Framework for Development of Electronic Healthcare Monitoring System using VPN Connection: International Journal of Advance Research in Computer Science and Management Studies: 1(7)

[17] Ogwueleka, F.N. 2010. Fundamentals of computer science: Printed by Okezzy productions.com

[18] Cheng-Kun, W. 2015. Security and privacy of personal health record, electronic medical record and health information: Problems and Perspectives in Management, 13(4)

[19] Li, Z. R., Chang, E. C., Huang, K. H., and Lai, F. 2011. A secure electronic medical record sharing mechanism in the cloud computing platform. Proceedings of the $15^{\text {th }}$ International Symposium on Consumer Electronics (ISCE'11) of the Institute of Electronics and Electrical Engineers (IEEE) Singapore 2011, 98-103 